\title{
Anti-Met/EGFR Monoclonal Antibody LY3164530
}

National Cancer Institute

\section{Source}

National Cancer Institute. Anti-Met/EGFR Monoclonal Antibody LY3164530. NCI

Thesaurus. Code C118281.

A monoclonal antibody (MoAb) against human epidermal growth factor receptor (EGFR) and human hepatocyte growth factor receptor (HGFR or c-Met), with potential antineoplastic activity. Upon administration, anti-Met/EGFR MoAb LY3164530 targets and prevents the activation of EGFR and c-Met. This leads to a downstream inhibition of EGFR/c-Met-mediated signal transduction pathways, and prevents cellular proliferation in tumor cells overexpressing EGFR and c-Met. EGFR, a member of the epidermal growth factor family of extracellular protein ligands, may be overexpressed on the cell surface of various solid tumor cell types. c-Met, a receptor tyrosine kinase overexpressed or mutated in many tumor cell types, plays a key role in cancer cell growth, survival, angiogenesis, invasion, and metastasis. 